Revista de Comunicación y Salud, 2018, Vol. 8, no 1, pp. 1-9

Editado por Cátedra de Comunicación y Salud

ISSN: 2173-1675

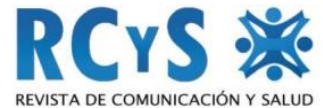

Enviado 26 de enero de 2018

Aprobado 7 de abril de 2018

\title{
PROPUESTA DE UN PLAN DE COMUNICACIÓN EN SALUD DIRIGIDO A ADULTOS MAYORES DIABÉTICOS TIPO 2
}

\section{Proposal of a health communication plan directed to type 2 diabetic senior adults}

María de la Caridad Casanova Moreno ${ }^{1 *}$, Héctor Demetrio Bayarre $\mathrm{Vea}^{2}$, Giselda Sanabria Ramos ${ }^{2}$, Daysi Antonia Navarro Despaigne ${ }^{3}$, Maricela Trasancos Delgado ${ }^{4}$

${ }^{1}$ Departamento provincial de Promoción y Educación para la Salud. Cuba

${ }^{2}$ Escuela Nacional de Salud Pública. Cuba

${ }^{3}$ Instituto Nacional de Endocrinología y Enfermedades Metabólicas. Cuba

${ }^{4}$ Hospital General Docente "Abel Santamaría Cuadrado". Cuba

\section{Resumen}

Introducción: La comunicación en salud nos ha demostrado ser de vital importancia para conseguir cambios en los comportamientos individuales y sociales de las personas. Objetivo: diseñar un plan de comunicación en salud dirigido a adultos mayores con diabetes mellitus para que obtengan conocimientos que le permitan vislumbrar la importancia de conocer los beneficios de los comportamientos saludables para un mejor control de la enfermedad. Método: se realizó una investigación de desarrollo tecnológico, se emplearon métodos del nivel teórico y del nivel empírico, en el Policlínico universitario "Hermanos Cruz" de Pinar del Río, en el primer trimestre de 2013. Resultados: se cometió el diagnóstico de la situación, se establecieron los comportamientos saludables que debía seguir la audiencia, se formularon los objetivos de conocimiento y comportamientos saludables e igualmente se realizó la definición de los mecanismos de monitoreo y evaluación. Conclusiones: se elaboró y propuso un plan de comunicación en salud dirigido a adultos mayores con diabetes mellitus, teniendo en cuenta los objetivos de conocimiento y comportamientos saludables, ofreciendo apreciando la importancia del mismo para la educación a adultos mayores con diabetes mellitus. El eslogan de los mensajes permite cumplir con el objetivo comportamental y de conocimiento que posee el mismo a partir de las actividades propuestas.

Palabras clave: diabetes mellitus; diabetes mellitus tipo 2, enfermedades no transmisibles, comunicación para la salud.

\footnotetext{
${ }^{1}$ Autor para correspondencia: María de la Caridad Casanova Moreno mcasanovamoreno@infomed.sld
} 


\begin{abstract}
Introduction: health communication has proved essential for changes in the individuals' own and social behaviours. Objective: to design a health communication plan directed to senior adults with diabetes mellitus, in order for them to obtain knowledge permitting them to notice the importance of knowing the benefits of healthy behaviours for better control of the disease. Methods: technologic development research using methods of the theoretical and empirical levels, carried out at Hermanos Cruz University Outpatient Polyclinic of Pinar del Río, in the first quarter of 2014. Results: the situation diagnosis was completed, the healthy behaviours were established to be followed by the audience, the objectives of knowledge and healthy behaviours were formulated and also the monitoring and assessment mechanisms were defined. Conclusions: a health communication plan was elaborated and proposed to be directed to senior adults with diabetes mellitus, considering the objectives of knowledge and healthy behaviours and appreciating the plan's importance for diabetes mellitus education of senior adults. The messages' slogan permits fulfilling, from the activities proposed, the latter's behavioural and knowledge-related objectives.
\end{abstract}

Keywords: diabetes mellitus, type 2 diabetes mellitus, non-communicable diseases, health communication.

\title{
Cómo citar el artículo
}

Casanova Moreno, M. C.; Bayarre Vea, H. D.; Sanabria Ramos, G.; Navarro Despaigne, D. A., y Trasancos Delgado, M. (2018). Propuesta de un plan de comunicación en salud dirigido a adultos mayores diabéticos tipo 2. Revista de Comunicación y Salud, 8(1), pp. 1-9. DOI: http://doi.org/10.35669/revistadecomunicacionysalud.2018.8(1).1-9

\section{INTRODUCCIÓN}

Las enfermedades no transmisibles (ENT) incluyen a las "cuatro grandes": enfermedad cardiovascular, cáncer, diabetes y enfermedad respiratoria crónica. Según la Organización Mundial de la Salud (OMS), las enfermedades no transmisibles fueron responsables del $63 \%$ de todas las muertes que se produjeron en el mundo en el año 2008 , concentrándose más de un $80 \%$ en los países en vías de desarrollo. Todas estas muertes se atribuyen, en gran medida, a una mala alimentación, actividad física insuficiente, uso de tabaco y uso excesivo de alcohol. Para aumentar la consciencia pública sobre las enfermedades no transmisibles y para apoyar su prevención y los esfuerzos de control, las Naciones Unidas (NU), la OMS, jefes de estado, ministros de salud y otros representantes de gobierno han hecho un llamado a una acción multisectorial y al compromiso de las diferentes partes interesadas (Fernstromet al., 2012).

Las partes interesadas deberían desarrollar una "cultura del bienestar" y alentar a los consumidores a pensar sobre la forma de obtener una dieta sensata y equilibrada, un estilo de vida activo y un peso saludable y deben realizar esfuerzos concertados para 
Propuesta de un plan de comunicación en salud dirigido a adultos mayores diabéticos tipo 2

enseñar al público la forma de evaluar la información de salud y deben comenzar las comunicaciones sobre salud en las primeras etapas de la vida (Fernstromet al., 2012).

Las serias limitaciones económicas, el bajo nivel educativo y el nulo o escaso acceso a información relativa a la salud son entre otros, factores determinantes que inciden directa 0 indirectamente sobre el estado de salud de las personas, familias y comunidades. Como todos sabemos, la desinformación o desconocimiento sobre promoción de la salud, prevención de la enfermedad, manejo y control de las enfermedades, impide la adopción de estilos de vida saludables. A través de la comunicación para la salud definida como "el proceso de presentar y evaluar información educativa persuasiva, interesante y atractiva que dé como resultado comportamientos individuales y sociales sanos", se busca facultar a las personas para que aumenten el control que tienen sobre salud y la forma de mejorarla (Fundación SERSOCIAL, 2016).

Las intervenciones directas en salud para mejorar la oferta de atención e incrementar la demanda por servicios no son suficientes para asegurar la sostenibilidad de los cambios logrados. La comunicación en salud nos ha demostrado ser de vital importancia para conseguir que estos cambios se mantengan e incluso se incrementen en el tiempo (Díaz Bordenave, 2012).

El cruce de la comunicación y la salud se constituyó como campo de estudio en las últimas décadas del siglo XX. En los países anglosajones, que han sido los impulsores a nivel académico de este campo de estudio, las investigaciones se desarrollaron en torno al concepto de health communication. En cambio, en el ámbito iberoamericano se carece todavía de un concepto único y se utilizan diferentes expresiones, como "comunicación y salud"; "comunicación para la salud o "comunicación en salud". De todos ellos, el de "comunicación y salud" parece ser el que se ha ido imponiendo en el ámbito académico, como punto de cruce entre esos dos ámbitos de investigación (Díaz, 2014).

A través del Plan de Comunicación, se fortalecen los programas de Educación Comunitaria para la Salud, Inducción a la Demanda de Servicios de Salud y Participación Social en Salud, se construyen y aplican herramientas comunicativas que, de una forma lúdica, didáctica, animada y con un lenguaje acorde al entorno cultural de los beneficiarios, empoderan a las comunidades más vulnerable de la región Caribe, sobre las estrategias y mecanismos con que cuentan para mantenerse saludables (Fundación SERSOCIAL, 2016).

Por todo lo expresado surge la necesidad de realizar un plan de comunicación en salud dirigido a adultos mayores diabéticos en el municipio Pinar del Río durante el año 2013.

\section{OBJETIVO}

Elevar el nivel de conocimiento de los mismos en cuanto a su enfermedad y la prevención de las complicaciones para obtener comportamientos saludables a mediano y largo plazo. 


\section{MÉTODO}

Para la realización del presente estudio se ha llevado a cabo una búsqueda sistemática en diferentes bases de datos, de ciencias sociales y de salud como son: Pubmed-Medline, Psyclnfo, Scielo, ISOC, Dialnet. Los descriptores utilizados fueron: "diabetes mellitus" AND "diabetes mellitus tipo 2" AND "enfermedades no transmisibles" AND "comunicación para la salud".

Se realizó una investigación de desarrollo tecnológico, se emplearon métodos del nivel teórico y del nivel empírico, en el Policlínico universitario "Hermanos Cruz" del municipio Pinar del Río en la provincia del mismo nombre, en el primer trimestre de 2013.

\section{RESULTADOS}

Plan de comunicación en salud dirigido a adultos mayores diabéticos

4.1. Título: Aprendiendo sobre la diabetes

4.2. Introducción: La comunicación es una herramienta imprescindible de la promoción de salud. La comunicación social en salud es un proceso planificado y orientado a promover en las personas la adaptación de nuevos comportamientos, debe fundamentarse en el modelo interactivo participativo.

4.3. Diagnóstico de la situación: Se identificaron los comportamientos no saludables para la audiencia y se realizó el esclarecimiento de los beneficios de un cambio de estos.

4.4. Propuesta de salud: Se establecieron los comportamientos saludables que debía seguir la audiencia.

4.5. Formulación de objetivos: Se formularon los objetivos de conocimiento y comportamientos saludables.

4.5.1. Objetivo comportamental: Que la población adulta mayor diabética tipo 2 obtenga comportamientos saludables.

4.5.2. Objetivo de conocimiento: Que la población adulta mayor diabética tipo 2 obtenga conocimientos que le permitan vislumbrar la importancia de conocer los beneficios de los comportamientos saludables para un mejor control de la diabetes.

4.6. Formulación de mensajes:

4.6.1. Conceptualización: El proyecto de los mensajes para los medios masivos de comunicación y para la comunicación interpersonal y grupal se respaldó, principalmente, en algunos elementos de la teoría del Aprendizaje Social de Bandura (González Valcárcel, B., 2006), del enfoque histórico cultural y de la actividad de Vigotsky (Vygotsky, Daniel H., 2003). Se asumió al mismo tiempo, la subjetividad (conocimiento de riesgo) y la capacidad conductual (auto eficacia).

4.6.2. Área de formación creativa: Demanda al área afectiva, cognitiva y conativa, papel de la familia en el apoyo al cambio de comportamiento, diseño de los pasos a seguir para el acogimiento de una conducta saludable, circunscribiendo dificultades a afrontar, el rol del grupo social

Revista de Comunicación y Salud, 2018, Vol. 8, no1, pp. 1-9 
Propuesta de un plan de comunicación en salud dirigido a adultos mayores diabéticos tipo 2

para la admisión y aprobación de una nueva conducta y elevación del conocimiento sobre el riesgo.

4.6.3. Eslogan para los mensajes: "Si controlamos nuestra diabetes, estaremos más sanos, productivos y felices"

Unificación de los medios de comunicación: Se renoció a la televisión municipal y la radio local como los medios de comunicación más efectivos para emitir la propuesta de mensajes de salud, por la accesibilidad, periodicidad de transmisión, predilección por la población.

4.7. Segmentación de la audiencia:

4.7.1. Audiencia primaria: población adulta mayor con DM2.

4.7.2. Audiencia secundaria: familia, médicos y enfermeras de la familia, Poder Popular, FMC, CDR.

4.8. Definición de los mecanismos de monitoreo y evaluación: Se trazó cómo efectuar el control constante de las actividades propuestas, así como la evaluación del proceso y efectividad. Igualmente se identificaron los mecanismos que se iban a seguir para verificar los disímiles pasos del plan de comunicación social en salud. Los educadores fueron los profesionales capacitados en el curso. Seguidamente, se refieren las actividades que dan salida a los objetivos, así como los criterios, indicadores y estándares.

4.9. Plan de comunicación en salud dirigido a adultos mayores con DM2.

\begin{tabular}{|c|c|c|c|c|c|}
\hline Actividad & $\begin{array}{c}\text { Criterio } \\
\text { evaluativo }\end{array}$ & Indicador & Estándar & $\begin{array}{l}\text { Método de } \\
\text { verificación }\end{array}$ & Instrumento \\
\hline $\begin{array}{l}\text { 1. Seminarios } \\
\text { sobre la } \\
\text { metodología de } \\
\text { la estrategia } \\
\text { educativa }\end{array}$ & $\begin{array}{l}\text { Participación de } \\
\text { los profesionales } \\
\text { de la salud y } \\
\text { líderes } \\
\text { administrativos y } \\
\text { formales en los } \\
\text { seminarios }\end{array}$ & $\begin{array}{l}\text { No. profesionales de } \\
\text { la salud y líderes } \\
\text { administrativos y } \\
\text { formales preparados / } \\
\text { Total } \\
\text { personas } \\
\text { comprendidas en } \\
\text { ambas } \\
\text { denominaciones } \times 100\end{array}$ & $90 \%$ & Observación & $\begin{array}{l}\text { Registro de } \\
\text { asistencia }\end{array}$ \\
\hline $\begin{array}{l}\text { 2. Discusión } \\
\text { grupal por un } \\
\text { mejor control de } \\
\text { la diabetes, en } \\
\text { el Consejo } \\
\text { Popular y } \\
\text { Consejo de } \\
\text { Salud }\end{array}$ & $\begin{array}{l}\text { Incluido en el } \\
\text { orden del día de } \\
\text { las agendas de } \\
\text { estas } \\
\text { organizaciones }\end{array}$ & $\begin{array}{l}\text { No. de reuniones } \\
\text { donde se trata el tema } \\
\text { /Total de reuniones } \\
\text { efectuadas } \times 100\end{array}$ & $70 \%$ & Inspección & $\begin{array}{l}\text { Guía de } \\
\text { inspección }\end{array}$ \\
\hline $\begin{array}{l}\text { 3. Diseño, } \\
\text { validación } \\
\text { y producción de } \\
\text { materiales } \\
\text { educativos } \\
\text { dirigidos a } \\
\text { personas } \\
\text { adultas mayores } \\
\text { con diabetes } \\
\text { mellitus }\end{array}$ & $\begin{array}{l}\text { Realización de } \\
\text { validación de } \\
\text { materiales } \\
\text { educativos }\end{array}$ & $\begin{array}{l}\text { No. de materiales } \\
\text { gráficos } \\
\text { producidos / No. de } \\
\text { materiales gráficos } \\
\text { planificados x } 100\end{array}$ & $100 \%$ & Inspección & $\begin{array}{l}\text { Guía de } \\
\text { inspección }\end{array}$ \\
\hline
\end{tabular}

Revista de Comunicación y Salud, 2018, Vol. 8, nำ1, pp. 1-9 
Propuesta de un plan de comunicación en salud dirigido a adultos mayores diabéticos tipo 2

\begin{tabular}{|l|l|l|l|l|l|}
\hline 4. Emisión radial & $\begin{array}{l}\text { Salida al aire de } \\
\text { los programas } \\
\text { fijos de radio que } \\
\text { abordan la } \\
\text { temática }\end{array}$ & $\begin{array}{l}\text { No. de programas } \\
\text { fijos de radio que } \\
\text { abordan la temática / } \\
\text { No. de } \\
\text { programas fijos de } \\
\text { radio } \\
\text { producidos x 100 }\end{array}$ & $100 \%$ & $\begin{array}{l}\text { Llamadas } \\
\text { telefónicas y } \\
\text { audiencia del } \\
\text { programa } \\
\text { como } \\
\text { radio } \\
\text { escucha }\end{array}$ & $\begin{array}{l}\text { Quejas y } \\
\text { sugerencias }\end{array}$ \\
\hline $\begin{array}{l}\text { 5. Emisión } \\
\text { televisiva } \\
\text { de mensajes } \\
\text { educativos }\end{array}$ & $\begin{array}{l}\text { Salida al aire de } \\
\text { los programas } \\
\text { fijos de televisión } \\
\text { (TV) que abordan } \\
\text { la temática }\end{array}$ & $\begin{array}{l}\text { No. de programas } \\
\text { fijos de TV que } \\
\text { abordan la temática / } \\
\text { No. de } \\
\text { programas fijos de TV } \\
\text { producidos x 100 }\end{array}$ & $100 \%$ & $\begin{array}{l}\text { Llamadas } \\
\text { telefónicas y } \\
\text { audiencia del } \\
\text { programa } \\
\text { como } \\
\text { televidente }\end{array}$ & $\begin{array}{l}\text { Registro de } \\
\text { llamadas }\end{array}$ \\
\hline
\end{tabular}

4.9.1. Cronograma de ejecución:

\begin{tabular}{|l|l|l|l|l|}
\hline \multicolumn{1}{|c|}{ Actividades } & \multicolumn{1}{|c|}{ Fecha } & \multicolumn{1}{|c|}{ Ejecutante } & Responsable & \multicolumn{1}{|c|}{ Lugar } \\
\hline $\begin{array}{l}\text { 1. Elaboración } \\
\text { de boletines } \\
\text { informativos }\end{array}$ & Anual & $\begin{array}{l}\text { Grupo de } \\
\text { trabajo }\end{array}$ & $\begin{array}{l}\text { Investigadora } \\
\text { principal }\end{array}$ & $\begin{array}{l}\text { Departamento Provincial } \\
\text { Promoción y Educación para la } \\
\text { salud. Escenarios } \\
\text { comunitarios }\end{array}$ \\
\hline $\begin{array}{l}\text { 2. Elaboración } \\
\text { de una } \\
\text { colección de } \\
\text { fotos }\end{array}$ & Anual & $\begin{array}{l}\text { Grupo de } \\
\text { trabajo }\end{array}$ & $\begin{array}{l}\text { Investigadora } \\
\text { principal }\end{array}$ & $\begin{array}{l}\text { Departamento Provincial } \\
\text { Promoción y Educación para la } \\
\text { salud. Escenarios } \\
\text { comunitarios }\end{array}$ \\
\hline $\begin{array}{l}\text { 3. Producción } \\
\text { de plegables y } \\
\text { guías } \\
\text { educativas }\end{array}$ & Anual & $\begin{array}{l}\text { Grupo de } \\
\text { trabajo }\end{array}$ & $\begin{array}{l}\text { Investigadora } \\
\text { principal }\end{array}$ & $\begin{array}{l}\text { Consulta multidisciplinaria } \\
\text { De atención integral al } \\
\text { diabético. }\end{array}$ \\
\hline $\begin{array}{l}\text { 4. Emisión } \\
\text { radial }\end{array}$ & Bimensual & $\begin{array}{l}\text { Grupo de } \\
\text { trabajo }\end{array}$ & $\begin{array}{l}\text { Investigadora } \\
\text { Principal }\end{array}$ & Emisora Radio Guamá. \\
\hline $\begin{array}{l}\text { 5. Emisión } \\
\text { televisiva de } \\
\text { mensajes } \\
\text { educativos }\end{array}$ & Bimensual & $\begin{array}{l}\text { Grupo de } \\
\text { trabajo }\end{array}$ & $\begin{array}{l}\text { Investigadora } \\
\text { principal }\end{array}$ & $\begin{array}{l}\text { Tele centro “Tele Pinar". } \\
\text { Programa de bien } \\
\text { público: Salud en Casa }\end{array}$ \\
\hline $\begin{array}{l}\text { 6. Barrios } \\
\text { debate }\end{array}$ & Trimestral & $\begin{array}{l}\text { Grupo de } \\
\text { trabajo }\end{array}$ & $\begin{array}{l}\text { Investigadora } \\
\text { Principal }\end{array}$ & Circunscripciones \\
\hline $\begin{array}{l}\text { 7. Videos- } \\
\text { debates }\end{array}$ & Trimestral & $\begin{array}{l}\text { Grupo de } \\
\text { trabajo }\end{array}$ & $\begin{array}{l}\text { Investigadora } \\
\text { principal }\end{array}$ & Sala de video Hermanos Cruz \\
\hline
\end{tabular}

\section{DISCUSIÓN Y CONCLUSIONES}

El plan de comunicación es una herramienta fundamental con la que hay que contar de forma ineludible a la hora de desarrollar la estrategia de comunicación de una institución sanitaria (ya sea laboratorio, clínica, asociación, ONG, farmacia o cualquier otra entidad relacionada).

La necesidad de un buen plan de comunicación es incuestionable hoy en día, pues en la era de las nuevas tecnologías y las redes sociales, que están transformando de forma radical al sector sanitario, una empresa que no comunica lo que hace es como si 
Propuesta de un plan de comunicación en salud dirigido a adultos mayores diabéticos tipo 2

no existiera. La visibilidad que proporciona la comunicación puede repercutir, además, de forma muy positiva en la reputación de la empresa (on y off line) (Comunicación en Salud, un blog de COM SALUD, 2014).

La importancia de la comunicación en el ámbito de la salud no se discute, y saber manejar las implicaciones emocionales, sociales y terapéuticas de la comunicación nos permite establecer relaciones altamente satisfactorias y productivas entre la población y los profesionales sanitarios (Pascual Segovia, 2012).

Existe una relación intrínseca entre salud y comunicación a partir de la perspectiva de la medicina familiar y comunitaria. Epistemológicamente se considera necesario reflexionar sobre dicho vínculo en el entendido de que generalmente se toman ambos conceptos de forma externa, lo que implica instrumentalizar la comunicación y simplificar el servicio ofrecido por los agentes sanitarios. Al considerar la atención en el primer nivel de salud como principal actividad, la comunicación es valorada como una experiencia de encuentros entre subjetividades, y su gestión dialógica pasa a ser crucial para conceptualizar de otra manera la misma noción de salud. La comunicación en salud es abordada, por tanto, como un elemento sanitario más, al tiempo que la salud muestra su aspecto comunicacional desde el punto de vista de las dinámicas culturales y los procesos de subjetivación (Álvarez Pedrosian, 2013).

La comunicación entendida como los procesos a través de los cuales las personas y los grupos dan sentido a su realidad, es una dimensión fundamental para el diseño y gestión de políticas intersectoriales de salud que actúen sobre los determinantes sociales de la salud (Díaz, 2012). Y lo es por dos razones:

- En primer lugar, porque facilita la articulación entre organismos y estamentos que históricamente no han participado en las políticas de salud. Si la perspectiva de determinantes sociales de la salud incide en el hecho de que todos somos, de alguna manera, productores de salud, un primer paso es convencer a los diferentes actores institucionales y sociales del protagonismo que deben asumir en ese camino. $Y$ para ello es necesario promover procesos de diálogo y articulación entre diferentes áreas de gestión, para alcanzar miradas comunes que permitan intervenir de forma sinérgica sobre la realidad. En ese sentido, la comunicación actúa en la construcción de una perspectiva integradora.

- En segundo lugar, porque la comunicación promueve la participación de los diferentes actores institucionales y sociales con capacidad de influir en la salud de la población. Hablamos entonces de una perspectiva que atraviesa la sociedad de forma transversal, rompiendo las fronteras institucionales e incorporando a actores que tienen llegada directa a los diferentes grupos sociales. En este sentido, la comunicación actúa como una perspectiva transdisciplinar, que recupera al sujeto como actor político, hacedor de su realidad.

Las tecnologías de información y comunicación (TIC), como el teléfono, el internet, la radio y la televisión, son herramientas útiles para las instituciones de salud, el 
profesional, el paciente y el cuidador familiar en situación de cronicidad. Las TIC permite al profesional de enfermería fomentar el desarrollo de conocimientos y habilidades en las personas con enfermedades crónicas no transmisibles y sus cuidadores, así como formar redes sociales y de apoyo para facilitar un mejor proceso de adaptación frente a la enfermedad, a través de fuentes que le permiten recoger, sistematizar y difundir información con el fin de mejorar el nivel de conocimientos e inducir cambios hacia comportamientos y estilos de vida que favorecen la salud física y mental en la diada cuidador sujeto de cuidado. Lo anterior se fundamenta en la necesidad de apoyo a los cuidadores familiares y a las personas con enfermedad crónica no transmisibles, que requieren de un soporte social, entendido éste como un elemento esencial en la interacción de los seres humanos (Barrera-Ortiz, CarrilloGonzález;, Chaparro-Díaz, Afanador, et al., 2011); Campos de Aldana, Moya Plata, Mendoza Matajira, et al., 2014); Herrera, y Romero (2012).

El plan de comunicación propuesto ofrecerá resultados, tanto en el conocimiento, como en el comportamiento en cuanto a su enfermedad en los adultos mayores diabéticos. El eslogan de los mensajes permite cumplir con el objetivo comportamental y de conocimiento que posee el mismo a partir de las actividades propuestas.

\section{BIBLIOGRAFÍA}

Álvarez Pedrosian, E. (2013). La salud como comunicación: medicina familiar y comunitaria. Revista de Comunicación y Salud, 3(2), 21-31.

Barrera-Ortiz, L.; Carrillo-González, G. M.; Chaparro-Díaz, L.; Afanador, N. P.; SánchezHerrera, B. (2011). Soporte social con el uso de TIC para cuidadores familiares de personas con enfermedad crónica. Revista de salud pública, 13(3), 446-57.

Campos de Aldana, M. S.; Moya Plata D.; Mendoza Matajira, J. D.; Duran Niño, E. Y. (2014). Las enfermedades crónicas no transmisibles y el uso de tecnologías de información y comunicación: revisión sistemática. Revista cuidarte, 5(1), 661-9.

Comunicación en Salud. Un blog de COMSALUD. Preguntas fundamentales antes de abordar un plan de comunicación en Salud. Recuperado de http://comsalud.es/web/blog/2014/09/05/preguntas-abordar-plan-comunicacion-salud/

Díaz, H. A. (2014). Comunicación de Organizaciones No Lucrativas de la Salud en España: el estado de la cuestión. Revista de Comunicación y Salud, 4, 35-48.

Díaz, H. (2012). La comunicación en todas las políticas de salud. Revista de Comunicación y Salud, 2(2), 45-46.

Díaz Bordenave, J. (2012). Guía de planificación participativa de estrategias de comunicación en salud. Ministerio de Salud Pública y Bienestar Social. Dirección general de promoción de la salud. Departamento de comunicación social para el desarrollo de la salud. Recuperado de http://www.mspbs.gov.py/promociondelasalud/wpcontent/uploads/2012/09/guia_corta estrategias_de_comunicacion_salud-3.pdf

Fernstrom, M. H.; Reed, K. A.; Rahavi, E. B.; Dooher, C. C. (2012). Estrategias comunicacionales para ayudar a reducir la prevalencia de enfermedades no transmisibles: resumen de la cumbre inaugural mundial sobre alimentación y actividad física de la Fundación IFIC. Revista Nutrition Reviews, 70(5), 301-310. 
Propuesta de un plan de comunicación en salud dirigido a adultos mayores diabéticos tipo 2

Fundación SERSOCIAL (2016). Planes de comunicación para la salud. Cartagena de Indias, Colombia: Fundación SERSOCIAL. Recuperado de http://sersocial.org/fundaser_web/index.php?option=com_content\&view=article\&id=10 $0:$ comuniacion\&catid $=1$ :latest-news\&ltemid $=62$

González Valcárcel, B. (2006). Contenidos y metodología de educación para la salud. En: Educación para la Salud. Módulo 9 de la Maestría en Promoción y Educación para la Salud. La Habana: ENSAP.

Herrera A, Flórez I., Romero E y Montalvo A. (2012). Soporte social a cuidadores familiares de personas con enfermedad crónica en Cartagena. Revista Aquichan, 12(3), 286-297.

Pascual Segovia, M. J. (2012). Reseña: Comunicar y curar. Un desafío para pacientes y profesionales sanitarios. Revista de Comunicación y Salud, 13(1), 67-71.

Daniel H. (2003). Vygotsky y la pedagogía. España: Paidós Ibérica, S. A. 